\title{
Non-Linear Vibrational Normal Modes of Biomolecules
}

\author{
Stavros C. Farantos \\ Institute of Electronic Structure and Laser \\ Foundation for Research and Technology-Hellas \\ Iraklion 71110, Crete, Greece, and \\ Department of Chemistry \\ University of Crete, Iraklion 71110, Crete, Greece
}

\begin{abstract}
The vibrational harmonic normal modes of a molecule, which are valid at relatively low energies close to a potential minimum, are extended by periodic orbits to higher energies where anharmonicity and coupling of the degrees of freedom set in. The families of periodic orbits which emanate from the equilibria (minima and transition states) of a molecular potential energy function are located with multiple shooting methods and they are continued in energy. The method is well established for triatomic molecules, and recently it has been applied to biomolecules such as alanine dipeptide. Long lived localized trajectories associated with specific conformations and non-linear vibrational modes can be traced. The influence of a solvent like water to excited non-linear normal modes is examined.
\end{abstract}

\section{Introduction}

Molecular spectroscopy has seen significant advances in both frequency and time domain in the last decades [1]. Techniques such as stimulated emission pumping, dispersed fluorescence, and high resolution Fourier transform and laser spectroscopy have contributed to the detailed study of small polyatomic molecules [9]. Laser femtosecond spectroscopy and molecular beams $[1,17]$ have allowed spectroscopists and dynamicists to study isolated molecules and to follow a chemical reaction in real time, where bonds are broken and new ones are formed. Furthermore, spectroscopic methods for studying structural and dynamic properties of complex molecules such as 2D NMR and 2D optical spectroscopy utilizing multiple ultrafast coherent laser pulses have allowed the study of protein structure and dynamics and femtosecond solvation dynamics [4, 22, 30].

The established theoretical methods based on a normal mode description of molecular vibrations which are applied at energies close to the equilibrium point, are not valid for vibrationally highly excited molecules. The deviation from the harmonic approximation of the potential energy surface imposes the need for the construction of accurate potential functions that describe several and energetically accessible reaction channels. These are non-linear functions and the application of non-linear mechanics to investigate the dynamics of the molecule is necessary.

Apart from the computational challenges, polyatomic molecules unravel conceptual and physical interpretation problems. A result of the non-linear mechanical behaviour of a dynamical system at high energies is the simultaneous appearance of ordered motions and chaos, as well as the genesis of new type of motions via bifurcation phenomena. As a matter of fact, the progress of non-linear mechanics forces us to reexamine the mechanisms of the breaking and/or forming a single chemical bond as it happens in elementary chemical reactions. New assignment schemes which allow the classification of quantum states in a meaningful and useful way are required and such novel methods have indeed been developed thanks to the theory of periodic orbits (POs), their bifurcations [7, 29] and the semiclassical quantization theories [16].

Periodic orbits may be considered as the non-linear counterparts of the harmonic normal modes of a molecule. They evolve with the energy of the system or any other parameter in the Hamiltonian, bifurcate and produce new periodic orbits which portrait the resonances among the vibrational degrees of freedom. Generally, POs reveal the structure of phase space at different energies, particularly, the localization of energy at specific bonds [24].

Recently, we have applied the methods of locating POs developed for small molecules to biological molecules, such as peptides described with empirical potential functions [27]. Using the alanine dipeptide as a prototype system, we have shown how one can systematically trace regions in phase space where the trajectories stay localized in specific vibrational modes of a conformation or of a transition state. With continuation techniques we obtain families 
of periodic orbits for an extended energy range and we find elementary bifurcations such as Hamiltonian saddle-node and Hopf like [28, 15]. In this article, we present preliminary results for the influence of a solvent like water on the low energy conformations of alanine dipeptide excited to specific non-linear normal modes.

\section{Computational Methods}

To locate periodic orbits in a dynamical system is equivalent of finding the roots of the non-linear equations which describe the return of the trajectory to its initial point in phase space, $x^{*}(0)$, after the time period $T$.

$$
B\left[x^{*}(0) ; T\right]=x^{*}\left[T ; x^{*}(0)\right]-x^{*}(0)=0 .
$$

$x$ denotes the vector of the generalized coordinates and their conjugate momenta for a system of $N$ degrees of freedom, and $x_{0}=x(0)$, the values at time $t=0 . x(T)$ is obtained by integrating Hamilton's equations of motion

$$
\frac{d x(t)}{d t}=J \partial H[x(t)] \quad(0 \leq t \leq T),
$$

where $H$ is the Hamiltonian function, and $J$ is a $2 N \times 2 N$ dimension matrix which is used to define the symplectic symmetry of a Hamiltonian system

$$
J=\left(\begin{array}{rr}
0_{N} & I_{N} \\
-I_{N} & 0_{N}
\end{array}\right) .
$$

$0_{N}$ and $I_{N}$ are the zero and unit $N \times N$ matrices respectively. A matrix $M$ which has the symplectic property satisfies the relation $J M J^{+}=M$.

The common procedure to find the roots of Eqs (1) is by linearizing the equations and using iterative schemes. For two nearby trajectories

$$
x_{0}^{\prime}=x_{0}+\Delta x,
$$

the correction $\Delta x$ is obtained by

$$
\begin{aligned}
B\left(x_{0}^{\prime} ; T\right) & \approx B\left(x_{0} ; T\right)+\frac{\partial B}{\partial x_{0}} \Delta x=0, \\
B\left(x_{0} ; T\right)+\left[\frac{\partial x\left(T ; x_{0}\right)}{\partial x_{0}}-I_{2 N}\right] \Delta x & =0 .
\end{aligned}
$$

The matrix

$$
Z(T)=\frac{\partial x\left(T ; x_{0}\right)}{\partial x_{0}},
$$

is the Fundamental Matrix, which is evaluated by integrating the variational equations (VE)

$$
\dot{Z}(t)=J \partial^{2} H[x(t)] Z(t) .
$$

Thus, to perform one iteration in a Newton-Raphson procedure for example, we first integrate for time $T$ Hamilton's and variational equations

$$
\begin{aligned}
& \dot{x}(t)=J \partial H[x(t)] \\
& \dot{Z}(t)=J \partial^{2} H Z(t),
\end{aligned}
$$

with initial conditions

$$
\begin{aligned}
& x(0)=x_{0} \\
& Z(0)=I_{2 N} .
\end{aligned}
$$

Then, we solve the linear algebraic Eqs (5)

$$
\left[Z(T)-I_{2 N}\right] \Delta x=-B\left(x_{0} ; T\right) .
$$

Solving the VE helps not only to locate POs but also to calculate the Lyapunov exponents which determine the stability of a trajectory. Particularly, for a periodic orbit of period $T$ the fundamental matrix, $M=Z(T)$, is called monodromy matrix from the eigenvalues of which we can determine the stability of the trajectories around the periodic orbit. An initial displacement $\Delta x$ after $k$ periods will become $\Delta x(k T)=M^{k} \Delta x$. Therefore, the eigenvalues of the monodromy matrix, $\mu$, dictate the stability of the nearby trajectories in the linearized system. Usually, $\mu$ is written as

$$
\mu=\exp (\lambda T)
$$

For conservative Hamiltonian systems the eigenvalues of the monodromy matrix appear as complex conjugate pairs, $\left(\mu, \mu^{*}\right)$, and one pair is always equal to one [24]. When all eigenvalues lie on the unit complex circle the PO is stable. If one pair of eigenvalues lies on the real axis and out of the unit circle the orbit is single unstable, if two pairs lie on the real axis the PO is called double unstable, and so on. For systems with larger than two degrees of freedom it may happen four eigenvalues are out of the unit circle on the complex plane, $\left(\mu, \mu^{*}, \mu^{-1},\left(\mu^{*}\right)^{-1}\right)$. In this case we call the periodic orbit complex unstable.

Once we have located one member of the family of periodic orbits we can use continuation techniques [6] to find trajectories for different periods $T$. This is done by using $T$ as the control parameter. Usually, for small increments of $T$ linear extrapolation methods are sufficient. By varying $T$ and thus the energy, the eigenvalues of the monodromy matrix move on the unit complex circle collide and may come out of the unit circle rendering the PO unstable and vice versa. At every period $T$ for which one pair of eigenvalues becomes equal to one, then a bifurcation takes place and new POs are born $[7,10]$.

Powerful existence theorems for POs [3, 12] guarantee that the predicted bifurcations in the linearized system will also remain in the non-linear system. From each minimum of a molecular potential energy surface we expect at least 
$N$ stable families of periodic orbits, which are called principal or fundamental. They are associated to the $N$ normal modes of the molecule. At a saddle point, the normal modes with pure imaginary eigenvalues (in the $2 N$ dimension phase space) give birth to principal families with unstable periodic orbits. The number of unstable directions is equal to the rank of instability of the saddle point.

We use multiple shooting techniques and the algorithms and computer codes have been described in previous publications [25]. The challenge to extend these methods to biomolecules requires the adoption of new practices. We use cartesian coordinates and empirical force fields to describe the forces among the atoms. We have adopted the Molecular Mechanics suite of programs, TINKER [23], to our computer code for locating periodic orbits, POMULT [26], in order to calculate the potential and its first (in Hamilton's equations) and second (in variational equations) derivatives analytically.

\section{Results and Discussion}

As an example of what we learn from a periodic orbit analysis of a highly excited triatomic molecule we review a recent study of $\mathrm{HOCl}[14]$. A complete quantum mechanical calculation has been carried out for this molecule. Accurate high level quantum chemistry calculations have produced an analytical potential function valid for the complete nuclear configuration space. Then the nuclear Schrödinger equation is solved in Jacobi coordinates, the distance of $\mathrm{Cl}$ atom from the center of mass of $\mathrm{OH}, R$, the bond length of $\mathrm{OH}, r$, and the angle between the distances, $\gamma$, to produce hundreds of vibrational eigenstates. The eigenfunctions are visually examined to find out regularities and the degree of localization in the configuration space. As energy increases, the assignment becomes cumbersome since most of the wave functions show a complicated nodal structure. However, overtone states may appear regular at even very high energies, and thus, they become easily assignable. Most interesting is the normal mode overtones which lead the molecule to dissociation (or isomerization), and for $\mathrm{HOCl}$ this is the $R$ mode. It was found, that while initially the eigenfunctions are localized along the $R$ coordinate, at some energy they started to deviate from this route. Simultaneously, a new progression of eigenfunctions emerged which were localized and properly oriented towards to the dissociation channel.

In Figure 1 we show the overtone states of the $R$ mode plotted on the minimum potential energy path. The circles denote the energy of the eigenstates, and also the extension of wave functions (localization). We can see, that at energy of about $-0.5 \mathrm{eV}$ the initial normal mode series, $\left(0,0, v_{3}\right)$, diverges and a new series of eigenfunctions localized along the $R$ coordinate appears, $(0,0, x)_{D} \cdot v_{3}$ and $x$ are the num-
Figure 1. Minimum energy path for $\mathrm{HOCl}$ along the dissociation coordinate $R$; the potential is minimized in the other two degrees of freedom. The symbols indicate the energy and the extension of the wave functions in two overtone progressions of eigenstates $(0,0, v 3)$ and $(0,0, x)_{D}$, respectively (see the text).

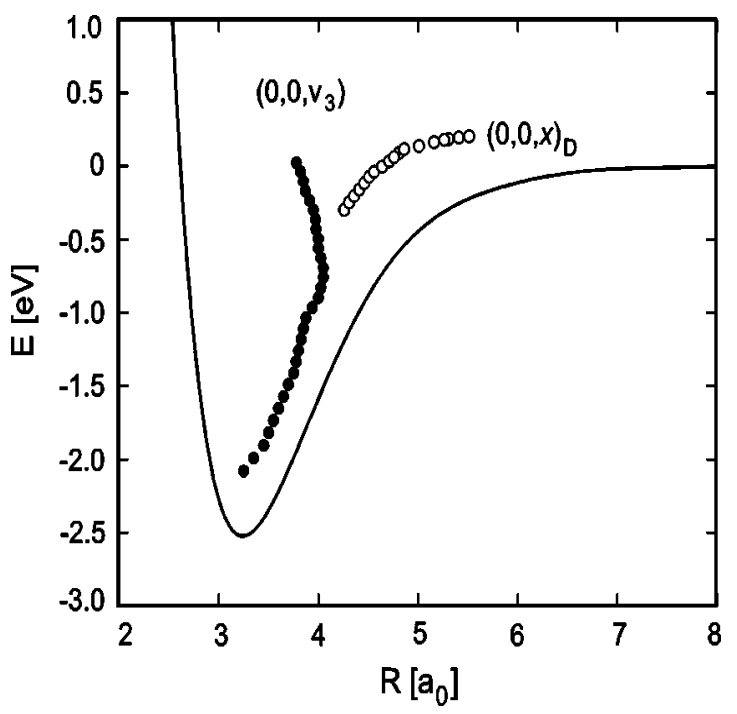

ber of quanta in the $R$ mode.

A similar analysis with periodic orbits is shown in Figure 2. The curves denote the energy of POs and the maximum extension in $R$. The principal family which corresponds to the $R$ stretch deviates at about $-0.5 \mathrm{eV}$ and a new family appears after a saddle-node bifurcation (SN). Saddle-node bifurcations appear abruptly at some energy, from which new periodic orbits emanate in two branches, one of them is usually with stable periodic orbits. Further analysis demonstrates the good correspondence among the new POs and overtone states. For more details we encourage the reading of the review articles [8, 18, 19].

Another approach to study non-linear phenomena in molecules is by employing spectroscopic Hamiltonians fitted to reproduce part of an experimental or theoretical spectrum. Then, by using Hamiltonian normal form expansion and semiclassical quantization the correspondence between classical and quantum mechanics is achieved $[13,11]$.

Biomolecules are complex systems, and therefore, it is not surprising that statistical mechanical methods are used for their study. The systematic methods of non-linear me- 
Figure 2. Minimum energy path of $\mathrm{HOCl}$ along the dissociation coordinate $R$; the potential is minimized in the other two degrees of freedom. The bold lines indicate the maximum extension of the periodic orbits in $R$. $\mathbf{R}$ denotes the principal family, and SN2 and SN saddle-node bifurcations, respectively (see the text).

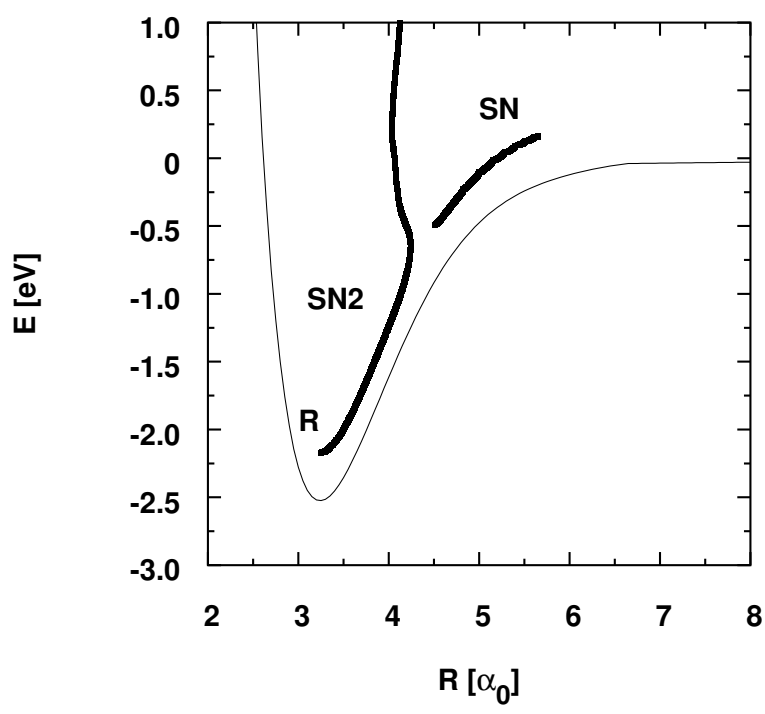

chanics based on hierarchically calculating stationary objects such as periodic orbits, tori and stable and unstable manifolds are considered only for systems with a few degrees of freedom. However, we argued before that periodic orbits offer the means to extract the physics from complicated calculations, and even to get reliable estimates of eigenenergies. Recently, we have demonstrated that periodic orbits can be located for biomolecules such as the dipeptide of alanine, a molecule with sixty internal degrees of freedom [27].

Alanine dipeptide has served as a prototype molecule for testing new algorithms in numerous studies in the past $[21,31]$. We also used this molecule by employing the parameters of CHARMM27 for the force field [20], Morse functions for the bond stretches and harmonic potentials for the angles. In this work, we investigated in detail the principal families emanating from the two lowest minima, $\min 1$, $\min 2$ and the transition state, $t s 1$, between them. The energy barrier for the lowest conformation $\min 1$ to isomerize to $\min 2$ is approximately $0.6 \mathrm{kcal} / \mathrm{mol}$ and the geometries of the two stable conformers are like those shown in Figures 6 and 7 to be discussed below. They differ in the orientation of left oxygen. The geometries of these two conformations correspond to a folded (absolute minimum) and to an elongated structure, respectively. Therefore, we use the distance of the two nitrogen atoms to assign trajectories trapped in one or the other minimum.

Among the sixty vibrational normal modes, we have chosen to study the $23 \mathrm{rd}$ and 24 th. The numbers used to assign the families are the same as the enumeration of the harmonic normal modes by increasing frequency. The $23 \mathrm{rd}$ and 24th normal modes have approximately localized motions. The $\mathrm{NH}$ and $\mathrm{CO}$ bonds oscillate in phase executing the largest displacements. Our interest to these particular normal modes came from their specificity. Starting with initial configurations from these oscillations and minimizing the energy we approach a specific minimum, the $f 23$ mode leads to $\min 1$ and the $f 24$ to $\min 2$.

In Figure 3 the continuation/bifurcation $(\mathrm{C} / \mathrm{B})$ diagram for the $f 23$ and $f 24$ families coming out from the three equilibria of the molecule $(\min 1, \min 2, t s 1)$ is shown. The principal families generated from minima are initially stable. The periodic orbits which emerge from the transition state start as unstable with the same rank of instability as the transition state. $t s 1$ has rank-1 instability. In this figure we plot the frequency obtained from the period of $\mathrm{PO}$ as a function of the total energy of the molecule. The anharmonic behaviour of the vibrational modes is evident. For the $f 24$ families of $\min 1$ and the saddle point, $t s 1$, an early saddle-node bifurcation is observed. This means that at a specific energy the continuation line levels off, decreasing its anharmonicity, and a new pair of families of periodic orbits emerge, one of them with stable periodic orbits and the other with unstable ones (we show the stable branch). The mechanism of appearance of such bifurcations has been described before [28]. It is worth noting the higher frequency of the 24th mode of $\min 1$ compared to the other two equilibrium points of the potential function. After the appearance of the saddle-node bifurcation (family $\min 1-f 24-s n 1)$ it was very difficult to continue this branch at higher energies. We expect a cascade of saddlenode bifurcations as we go up in energy [8].

The question of what happens to a polypeptide in water solution has been addressed by several investigators [32, 2]. Here, we examine the stability of $\min 1$ conformation excited to the normal mode $f 23$, for example, when it is embedded in water. This is done by choosing initial conditions for the coordinates and velocities of the alanine dipeptide atoms along the periodic orbits of a specific isomer and normal mode. Then, we combine them with the coordinates and velocities of 487 water molecules, described by the TIP3P parameters. These embedded states simulate the excitation of alanine dipeptide in an overtone state. After that, the dynamics is followed for fifty picoseconds in a canonical ensemble at several temperatures. 
Figure 3. The continuation/bifurcation diagram for alanine dipeptide for two normal modes, $f 23$ and $f 24$ of the three stationary points, $\min 1, \min 2$, and $t s 1$. The frequencies are calculated from the periods of the periodic orbits: $\omega=2 \pi \hbar / T$.

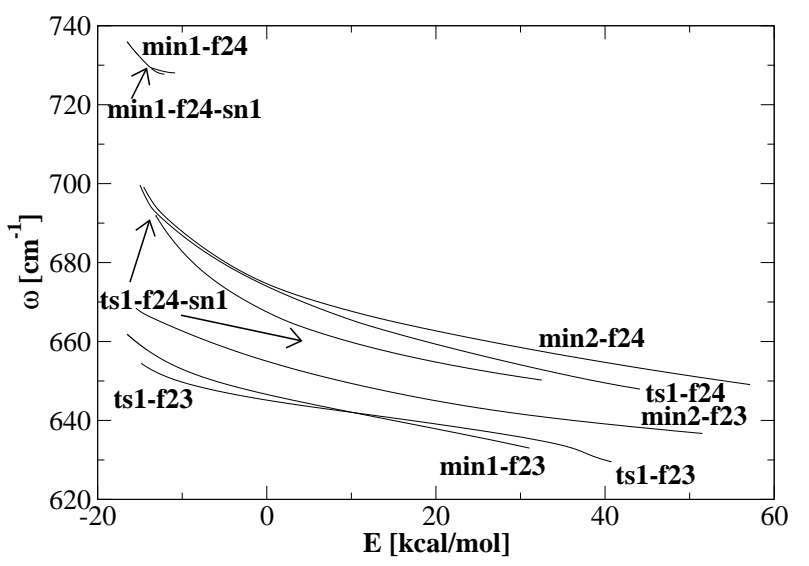

Embedded states obtained from the $\min 1-f 23 \mathrm{PO}$ and at energy $-7 \mathrm{kcal} / \mathrm{mol}$ are studied for several temperatures. We found that at $50 \mathrm{~K}$ the dipeptide preserves the folded structures. For temperatures 100 and $200 \mathrm{~K}$ trajectories are trapped to both minima with folded and unfolded structures, whereas at room temperature almost all trajectories quickly take the unfolded form. Representative trajectories at temperature $100 \mathrm{~K}$ are plotted in Figure 4 for times up to $2 \mathrm{ps}$, and in Figure 5 up to 50 ps. From Figure 4, we can see that the interaction of alanine dipeptide with water destabilizes the initial geometry of the molecule and in times less than $0.5 \mathrm{ps}$ the trajectory jumps to the unfolded configurations or remains into the folded one. Minimization of the energy for the total system (water and alanine dipeptide) results in lower energy minima with open geometries than closed ones. The preferred opens structures at room temperatures have been found in previous studies as well $[32,2]$. Here, we point out that folded isomers can also be selected and they survive for several picoseconds at low temperatures.

Figures 6 and 7 show configurations of the solution at $150 \mathrm{~K}$ with folded and unfolded dipeptide.

Localization in complex systems is currently a subject of intense research $[4,5]$. For example, energy localization and the theory of breathers have been utilized to argue for the existence of long, non-exponential excited state
Figure 4. The variation of the distance of the two nitrogen atoms in alanine dipeptide in 2 ps. Representative trajectories are shown from a run at $100 \mathrm{~K}$ and in a canonical ensemble.

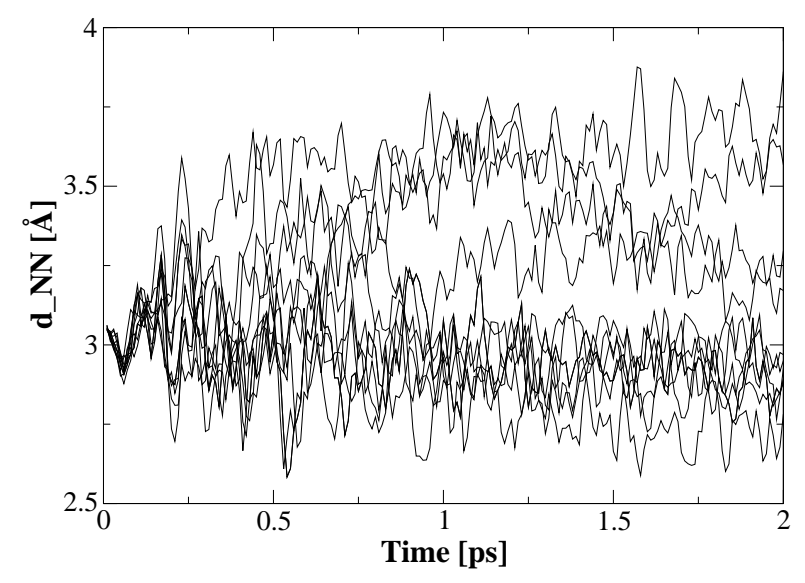

relaxation in myoglobin [4]. In these studies the authors used simple models to argue that localized states may be responsible for the observed long relaxation times. Our study unequivocally demonstrates the existence of stable periodic orbits for substantial energy ranges in alanine dipeptide described with an empirical potential function. Such potentials are widely used in simulations of biomolecules.

We find different time scales in the isomerization process depending on the excitation of specific vibrational modes but from different conformations. In spite of exciting similar modes in the three conformations their dynamics differ substantially. Controlling chemical reactions at such a level is one of the goals of chemical dynamics. However, novel spectroscopic methods have indeed appeared which study small peptides in subpicosecond time scale. In a recent investigation of alanine tripeptide in water by twodimensional vibrational spectroscopy, conformational fluctuations at the time scale of $0.1 \mathrm{ps}$ have been reported [30]. It is worth noting that the stability parameter of the unstable POs which originate from the transition state $t s 1$ gives an upper estimate of the lifetime of the complex. This time is predicted to be 0.3 ps close to that found in ref. [30]. 
Figure 5. The variation of the distance of the two nitrogen atoms in alanine dipeptide in 50 ps. Representative trajectories are shown from a run at $100 \mathrm{~K}$ and in a canonical ensemble.

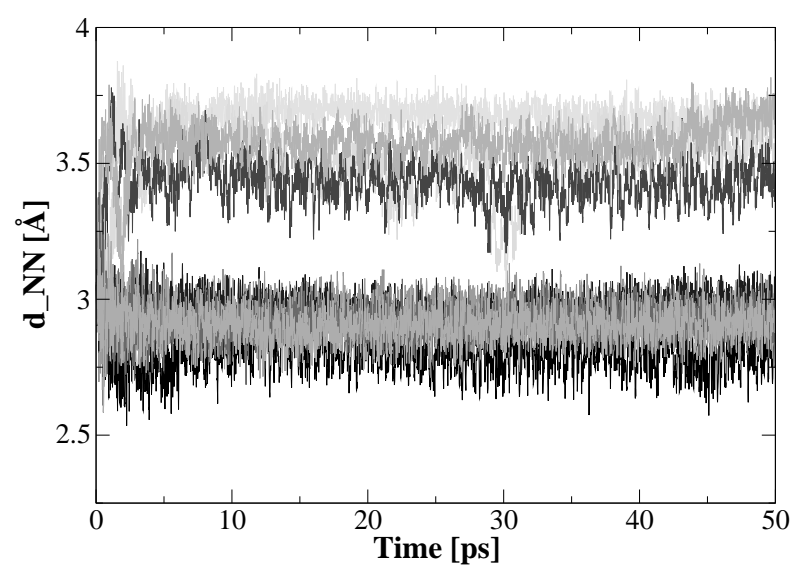

\section{Conclusions}

In chemical reactions, we expect that at energies where bonds dissociate and new ones are formed non-linearity will play a significant role. By locating periodic orbits we make the first approximation in understanding the complexity of the non-linear dynamics and predicting localization of energy in particular modes, and thus, selectivity. The development of novel spectroscopic methods for the study of isolated molecules and in solutions at subpicosecond times will increase the need for systematic theoretical investigations such as the periodic orbit analysis offers.

\section{Acknowledgments}

I am grateful to Dr. Reinhard Schinke and Dr. Sergy Grebenshchikov for stimulating discussions. Financial support from the Ministry of Education and European Union in the frame of the program Pythagoras (EPEAEK) is kindly acknowledged. This research was in parts supported by the European Union ToK grant (MTKD-CT-2005-029583),

\section{References}

[1] A. H. Zewail. "Femtochemistry: Atomic-Scale Dynamics
Figure 6. A typical folded structure of alanine dipeptide in water in a simulation with a canonical ensemble and at $150 \mathrm{~K}$. From left to right the tubes correspond to the atoms of the chemical structure $\mathrm{CH}_{3} \mathrm{CONHCH}\left(\mathrm{CH}_{3}\right) \mathrm{CONHCH}_{3}$.

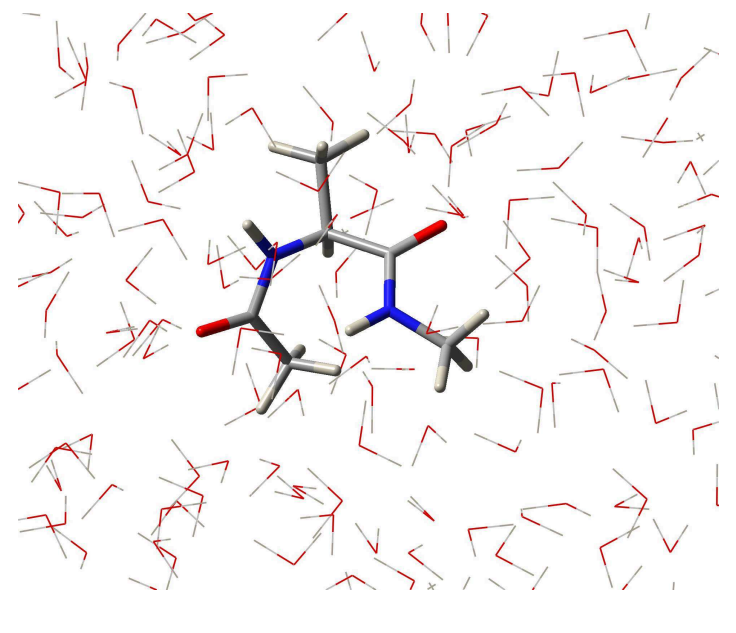

of the Chemical Bond". J. Phys. Chem. A, 104:5660, 2000.

[2] A. N. Drozdov, A. Grossfield, and R. V. Pappu. "Role of Solvent in Determining Conformational Preferences of Alanine Dipepted in Water". J. Am. Chem. Soc., 126:2574-2581, 2004.

[3] A. Weinstein. "Normal Modes for Non-Linear Hamiltonian Systems". Inv. Math., 20:47-57, 1973.

[4] A. Xie, L. van der Meer, W. Hoff, and R. H. Austin. "Longlived Amide I Vibrational Modes in Myoglobin". Phys. Rev. Lett., 84:5435-5438, 2000.

[5] D. K. Campbell, S. Flach, and Y. S. Kivshar. "Localizing Energy Through Nonlinearity and Discreteness". Physics Today, 43, January 2004.

[6] E. L. Allgower, and K. Georg. "Numerical Continuation Methods, Springer series in computational mathematics", volume 13. (Springer-Verlag, Berlin), 1993.

[7] H. Hanssmann. "Local and Semi-Local Bifurcations in Hamiltonian Dynamical Systems". Lecture Notes in Mathematics, Springer-Verlag, Berlin, 2007.

[8] H. Ishikawa, R. W. Field, S. C. Farantos, M. Joyeux, J. Koput, C. Beck, and R. Schinke. "HCP - CPH Isomerization: Caught in the Act”, volume 50. Ann. Rev. Phys. Chem., 1999.

[9] H.-L. Dai , and R.W. Field. "Molecular Dynamics and Spectroscopy by Stimulated Emission Pumping”. (World Scientific, Singapore), 1995.

[10] J. D. Crawford. "Introduction to Bifurcation Theory". Rev. Mod. Phys., 63:991-1037, 1991.

[11] J. Main, C. Jung, and H. S. Taylor. "Extracting the Dynamics in Classically Chaotic Quantum Systems: Spectral Analysis of the $\mathrm{HO}_{2}$ Molecule". J. Chem. Phys., 107:6577, 1997. 
Figure 7. A typical open structure of alanine dipeptide in water in a simulation with a canonical ensemble and at $150 \mathrm{~K}$. From left to right the tubes correspond to the atoms of the chemical structure $\mathrm{CH}_{3} \mathrm{CONHCH}\left(\mathrm{CH}_{3}\right) \mathrm{CONHCH}_{3}$.

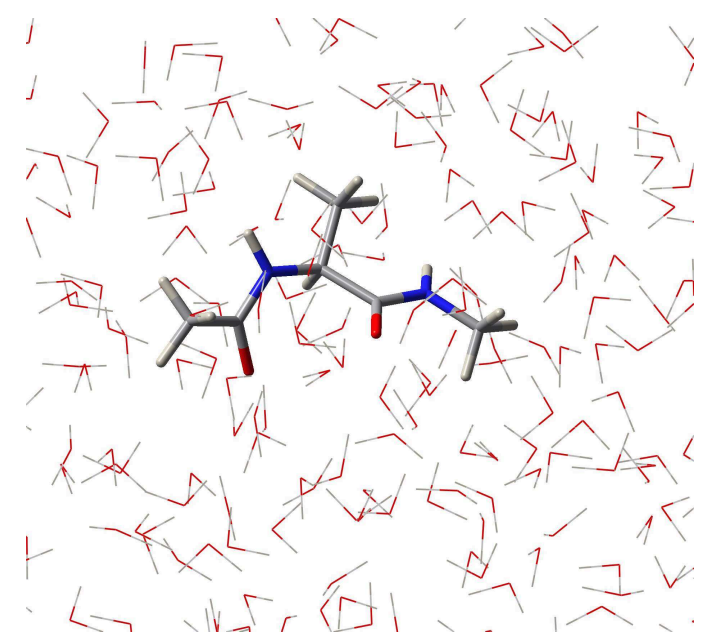

[12] J. Moser. "Periodic Orbits Near an Equilibrium and a Theorem by Alan Weinstein". Commun. Pure Appl. Math., 29:727-747, 1976.

[13] J. Svitak, Z. Li, J. Rose, and M. E. Kellman. "Spectral Patterns and Dynamical Bifurcation Analysis of Highly Excited Vibrational Spectra". J. Chem. Phys., 102:4340, 1995.

[14] J. Weish, J. Hauschildt, R. Düren, R. Schinke, J. Koput, S. Stamatiadis, and S. C. Farantos. Saddle-node Bifurcations and their Quantum Mechanical Fingerprints in the Spectrum of HOCl. J. Chem. Phys., 112:77, 2000.

[15] Jan-Cees van der Meer. "The Hamiltonian Hopf bifurcation”. Springer-Verlag, New York, 1985.

[16] M. C. Gutzwiller. "Chaos in Classical and Quantum Mechanics”, volume 1. (Springer-Verlag), 1990.

[17] M. Herman, J. Lievin, and J. V. Auwera. "Global and Accurate Vibration Hamiltonians from High-Resolution Molecular Spectroscopy". Adv. Chem. Phys., 108, 1999.

[18] M. Joyeux, S. C. Farantos, and R. Schinke. "Highly Excited Motion in Molecules: Saddle-Node Bifurcations and their Fingerprints in Vibrational Spectra". J. Phys. Chem., 106:5407, 2002.

[19] M. Joyeux, S. Yu. Grebenshchikov, J. Bredenbeck, R. Schinke, and S. C. Farantos,. Intramolecular Dynamics Along Isomerization and Dissociation Pathways, in "geometrical structures of phase space in multi-dimensional chaos". Adv. Chem. Phys., 130:267-303, 2005.

[20] N. Foloppe, and A. D. MacKerell, Jr. "All-Atom Empirical Force Field for Nucleic Acids: I. Parameter Optimization Based on Small Molecule and Condensed Phase Macromolecular Target Data". J. Comput. Chem., 21:86-104, 2000 .
[21] P. G. Bolhuis, C. Dellago, and D. Chandler. "Reaction Coordinates of Biomolecular Isomerization". Pros. Nat. Acad. Sci. USA, 97:5877, 2000.

[22] P. Hamm, M. Lim, W. F. DeGrado, and R. M. Hochstrasser. "The Two-Dimensional IR Nonlinear Spectroscopy of a Cyclic Penta-Peptide in Relation to its Three-Dimensional Structure". Proc. Natl. Acad. Sci. USA, 96:2036, 1999.

[23] J. Ponder. "Software Tools for Molecular Design". http://dasher.wustl.edu/tinker/, version 4.1, 2004.

[24] S. C. Farantos. Chemical Dynamics: A Periodic Orbits Approach. In J. Broeckhove, and L. Lathouwers, editor, "Time Dependent Quantum Mechanics: Experiments and Theory”, pages 27-43. (Plenum Co. Ltd.), 1992.

[25] S. C. Farantos. Methods for Locating Periodic Orbits in Highly Unstable Systems. THEOCHEM J. Mol. Struct., 341:91, 1995.

[26] S. C. Farantos. "POMULT: A Program for Computing Periodic Orbits in Hamiltonian Systems Based on Multiple Shooting Algotithms". Comp. Phys. Comm., 108:240, 1998.

[27] S. C. Farantos. "Periodic Orbits in Biological Molecules: Phase Space Structures and Selectivity". J. Chem. Phys., 126:175101, 2007.

[28] S. C. Farantos, Z.W. Qu, H. Zhu, and R. Schinke. "Reactions Paths and Elementary Bifurcations Tracks: the diabatic ${ }^{1} B_{2}$-state of ozone". Int. J. Bif. Chaos, 16:1913-1928, 2006.

[29] S. Wiggins. "Introduction to Applied Non-linear Dynamical Systems and Chaos". Second Edition (Springer-Verlag New-York, Inc.), 2003.

[30] S. Woutersen, Y. Mu, G. Stock, and P. Hamm. "Subpicosecond Conformational Dynamics of Small Peptides Probed by Two-Dimensional Vibrational Spectroscopy". Proc. Natl. Acad. Sci. USA, 98:11254-11258, 2001.

[31] W. Ren, E. Vanden-Eijnden, P. Maragakis, and Weinan E. "Transition Pathways in Complex Systems: Application of the Finite-Temperature String Method to the Alanine Dipeptide". J. Chem. Phys., 123:134109, 2005.

[32] Wen-Ge Han, K. J. Jalkanen, M. Elstner, and S. Suhai. "Theoretical Study of Aqueous N-Acetyl-L-Alanine-N'Methylalamide: Structures and Raman VCD, and ROA Spectra”. J. Phys. Chem. B, 102:2587-2602, 1998. 